\title{
Characterization and Mechanism Analysis of Alkali-Silica Reaction in Cementitious Materials
}

\author{
Qingli Dai, Professor \\ Civil and Environmental Engineering, Michigan Technological University \\ USA
}

\begin{abstract}
Alkali-silica reaction is a severe threat to the concrete infrastructure durability and its damage mechanism has not been fully understood currently. This thesis investigated the ASR damage mechanism from the multiphysical and kinetic-thermodynamic prospect. The objective of this study is to characterize the chemical and microstructural properties of synthesized and formed Alkali-Silica Reacted (ASR) gels, expansive microcrack morphology and to develop kinetic-thermodynamic frame work for ASR gel formation and related damage development. The chemical and microstructure characterization were conducted on synthesized gels (with different metal ratios) by using sophisticate neutron or X-ray scattering techniques. The chemical formulation and gel water content were measured based on neutron scattering intensity of different types of samples. The gel particle or pore size distribution was characterized with X-ray scattering and the SEM imaging techniques. Comparable size distributions were found among these measurements. The ASR gel formation and damage development were characterized with both florescent microscope and SEM images in cementitious material samples. The in-situ damage development and gel formation were also captured with dynamic X-ray CT techniques. The effects of different SCMs (glass power and fly ash) on ASR development reduction was also investigated. The alkali-silica reaction kinetics was measured with a model reactant test. The silica dissolution rate in simulated pore solution at different reaction stages were obtained. The calibrated reaction rates at different reaction stages were inputted into a kinetic-thermodynamic model for simulating phase development (including ASR gel formation) and pore solution concentration changes. The current simulation results showed the ASR gel formation mechanism and the predicted gel content and pore solution changes are consistent with measured results. Followed the kinetic-thermodynamic analysis of ASR gel formation, the fracture simulation was conducted with BEM-DDM scheme to predict the microcrack simulation inside of irregular particles and aggregates. The predicted microcrack development was favorably compared with the captured X-ray CT image data. In summary, this study increases the fundamental understanding of alkali-silica reaction kinetics and damage formation mechanisms at different reaction conditions. The ASR mitigation strategies can be developed in the future work based on these fundamental studies.
\end{abstract}

